\title{
Materialentwicklung für schulischen Unterricht - ein praxisorientierter Blick in einen multiprofessionellen Prozess
}

\begin{abstract}
Zusammenfassung
Nichtregierungsorganisationen (NRO), die sich der Gemeinnützigkeit verpflichtet haben, führen ihren Beitrag zur Erfüllung ihres gesellschaftlichen Auftrags auch mit Bildungsarbeit aus. Ihr Interesse ist es, aktuelle gesellschaftliche Themen aus zivilgesellschaftlicher Perspektive in den schulischen Unterricht zu bringen. Hierzu entwickeln sie zahlreiche und vielfältige Bildungsmaterialien. Im Gegensatz zu staatlich genehmigten Lehr- und Lernmaterialien müssen diese Materialien der NRO keinen (staatlichen) externen Prüfprozess durchlaufen, bevor sie im Unterricht zum Einsatz kommen können. Doch welchen spezifischen Mehrwert können NRO-Bildungsmaterialien für alle am Schulleben Beteiligten bieten? Nach welchen Prämissen wird dieses Material entwickelt? Und wie kann sichergestellt werden, dass die darin zum Ausdruck kommende spezifische Expertise von NRO möglichst anschlussfähig an das formale Bildungssystem ist? Diese Fragen werden in diesem Beitrag entlang des jüngst erschienenen Greenpeace-Bildungsmaterials „Heiße Zeiten - Klima und Gesellschaft im Wandel“, der Entwicklungsprozess und die darin ausgehandelten Interessen und Ansprüche analysiert.
\end{abstract}

Schlüsselworte: Materialentwicklung, Unterrichtsmaterial, BNE, NRO/NGO, Globales Lernern

\footnotetext{
Abstract

Non-governmental organizations (NGOs) which are committed to the benefit to the society, carry out their contribution to the fulfillment of their societal mission also in the field of education. Their interest often is to bring current societal issues into the classroom from a civil society perspective. Numerous and diverse educational materials are developed for this purpose. In contrast to state-approved teaching and learning materials, NGO materials do not have to go through a (state) external review process before they can be used in class. But what are the specific added value of teaching materials offered by NGOs for all members involved in schoollife. According to which premises this teaching material is developed? And how can it be ensured that the specific expertise of NGOs expressed in the material is compatible to the formal education system?
}

These questions are analyzed in this article as well as the development process and the interests and claims negotiated alongside the recently published Greenpeace educational material „Heiße Zeiten - Klima und Gesellschaft im Wandel“".

Keywords: material development, teaching material, ESD, NRO/ $N G O$, global learning

\section{Rahmensetzungen einer Kooperation von Schule und NRO}

Mit den komplexen globalen Herausforderungen wachsen auch die Herausforderungen, auf die Schulen mit ihrem Bildungsauftrag zeitnah und fachlich angemessen reagieren müssen. Komplexen Herausforderungen stehen oft komplexe Lösungsansätze gegenüber, die Kompetenzen, wie kritisches und systemisches Denken, aber auch Kreativität und Engagement voraussetzen. Kontroverser, debattenorientierter Unterricht ist hier insofern von großer Bedeutung, als dass Lernende in einem solchen Unterricht ihre individuellen Kompetenzen stärken können, die sie benötigen, um diese Herausforderungen verstehen sowie sachgerecht Entscheidungen treffen zu können und entsprechend handlungsfähig zu werden.

In den letzten Jahren lässt sich eine Zunahme an Bildungsangeboten von zivilgesellschaftlichen Organisationen beobachten, die ein solches Lernen explizit anregen wollen. Laut des ZiviZ-Survey 2017 ist Bildung das zweitgrößte Handlungsfeld zivilgesellschaftlicher Organisationen (ZiviZ-Survey, 2017, S. 7), wobei die meisten Kooperationen mit der Organisation Schule stattfinden. Das Konzept der offenen Schule, außerschulische Partner aktiv in Bildungsprozesse einzubinden, gibt NRO, wie beispielsweise Umweltschutz- oder entwicklungspolitischen Organisationen vielfältige Möglichkeiten, ihr spezifisches Themenspektrum aus der Perspektive der Zivilgesellschaft in Schule einzubringen und ihre Expertise so für Schule und Unterricht nutzbar zu machen (Moeller, 2009, S. 4). Als Potenzial wird dabei gesehen, dass NRO-Vertreter*innen ,in ihrer Rolle als engagierte und aktive BürgerInnen eine Vorbildfunktion für SchülerInnen“ übernehmen und „Handlungsmöglichkeiten der politischen Beteiligung 
unmittelbar erfahrbar" (Böhme, 2019, S. 436) machen können. Insofern überrascht es nicht, dass Kooperationen zwischen Schule und NRO mittlerweile kein Nischenthema mehr sind und von den Bildungsverwaltungen der Länder sogar explizit unterstützt werden (ebd., S. 436). Mit ihrer Hilfe können die Rahmenlehrpläne mit aktuellen gesellschaftlichen Themen ergänzt und insbesondere im Kontext einer Bildung für nachhaltige Entwicklung in globalen Zusammenhängen gefördert werden. In einigen Bundesländern werden Kooperationen zwischen Schulen und NRO auch von ministerialer Ebene systematisch gefördert: So werden zum Beispiel in der Rahmenvereinbarung zur Kooperation von Schule und entwicklungspolitischen Initiativen zwischen der Senatsverwaltung für Bildung, Wissenschaft und Forschung und dem Berliner Entwicklungspolitischen Ratschlag (BER e.V.) konkrete Formen der Zusammenarbeit benannt. Dazu zählt u.a. die Fortbildung von Lehrkräften und Erzieherinnen und Erziehern, die Umsetzung von Projekttagen oder -wochen sowie die Begleitung von Schulentwicklungsprozessen im Rahmen von Schulprogrammen oder die Mitgestaltung von Ganztagsschulen.

Auch Hessen praktiziert seit vielen Jahren im Bereich der Umweltbildung eine enge Kooperation zwischen zivilgesellschaftlichen Gruppen und dem offiziellen Fortbildungssystem des Landes. So führen 11 regionale unabhängige Bildungszentren für nachhaltige Entwicklung im Namen des hessischen Kultusministeriums Lehrerfortbildungen durch und beraten die Schulen bei der Konzeptentwicklung und Umsetzung. Für den Bereich Klima wurde ab 2018 gar ein eigener Schwerpunktbereich mit dem Portal https://www.klimabil dung-hessen.de/ startseite.html aufgebaut, in dem alle Fortbildungs- und Beratungsangebote gebündelt werden. In Baden-Württemberg bestehen vielfältige, wenn auch nicht in der gleichen Verzahnung gestaltete Kooperationen mit einer Vielzahl außerschulischer Lernorte, die im Kontext der Bildung für nachhaltige Entwicklung im landeseigenen BNE-Kompass (https://www.bne-kom pass.de/start/) gelistet werden. Unterhalb dieser Kooperationsebene von landesspezifischen Ministerien und NRO hat sich eine bunte Vielfalt intensiver Kooperationsnetzwerke entwickelt, die von kurzzeitigen oder langfristigen Projekten bis hin zu auf Dauer angelegte Maßnahmen unterschiedliche Angebote umsetzen. Ein Beispiel dafür ist die vielfältige Kooperation der im Herbst 2019 eröffneten KlimaArena in Hoffenheim mit unterschiedlichsten Schulen aus Hessen, Rheinland-Pfalz und Baden-Württemberg. Zudem wird im Orientierungsrahmen für den Lernbereich globale Entwicklung (KMK/BMZ, 2015) explizit auf die Bedeutung der Zusammenarbeit zwischen Schule und NRO hingewiesen. Diese kann im Sinne einer Aufgabe der ganzen Schule als fester Bestandteil im Schulprogramm verankert werden (ebd., S. 428).

Vor diesem Hintergrund stellt sich die Frage, wie die hier deutlich werdenden unterschiedlichen Formen der Kooperation einen entsprechenden Mehrwert für die am Schulleben Beteiligten entfalten und Wirkung zeigen. Die folgenden Ausführungen konzentrieren sich dabei auf die Bereitstellung von Lehr- und Lernmaterialien als Kooperationsform. In diesem Kontext ist zu fragen: Welchen schulischen Mehrwert bieten NGO-Bildungsmaterialien und wie kann sichergestellt werden, dass die darin zum Ausdruck kommende spezifische Expertise von NRO anschlussfähig für das formale Bildungssystem ist?

\section{Überlegungen zum potenziellen Mehrwert} von NRO-Materialien im Kontext Schule

Lehrkräfte wissen vor dem Hintergrund der Motivation ihrer Schülerinnen und Schüler oder deren Lebensweltbezug um die - wenn auch in verschiedenen Unterrichtsfächern ungleiche Relevanz aktueller Ereignisse als Herausforderung für die Konzipierung und Gestaltung von Unterricht. Dessen Bedeutung liegt vor allem darin, dass sich Schüler*innen angesichts aktueller, ergebnisoffener Ereignisse und Entwicklungen (etwa mit Blick auf den Klimawandel, die interkontinentalen Migrationsprozesse oder die Digitalisierung) in besonderen Lernsituationen befinden. Solche Lernsituationen sind zumeist stark von der allgemeinen Medienberichterstattung sowie von Aspekten, die in Social-Media-Kanälen diskutiert werden, dominiert. Entsprechend sehen die Schüler*innen sich gefordert, die aktuellen Entwicklungen für sich einordnen und bewerten zu müssen. Gleich, ob ein Vulkan ausgebrochen ist, neue Zoonosen wie Covid-19 mit gravierendem globalem Ausmaß auftreten, große Bereiche eines Landes durch Unwetter überflutet wurden oder Giftstoffe verheerende Umweltschäden verursachen, in all diesen Fällen stehen Fragen im Raum, wie mit solchen Szenarien umzugehen ist, welche Wirkungen sie für wen entfalten und ob und wie sie künftig vermieden werden können. Wenngleich hier Erfahrungswerte vorliegen, Katastrophenpläne erstellt wurden und Schutzmaßnahmen in vielen Fällen greifen, besteht für Schule die Herausforderung, solche Ereignisse im Unterricht qualitativ hochwertig thematisieren zu können. Hier bedarf es entsprechend valider Informationen und Unterrichtsmaterialien.

Schulbücher können einem entsprechenden Aktualitätsbezug nur bedingt gerecht werden. Bedingt deswegen, weil sie vergleichbare Ereignisse aus der Vergangenheit, etwa Naturereignisse oder sozioökonomische und politische Konflikte beispielhaft aufbereitet und die dahinterstehenden Strukturen und Prozesse grundsätzlich dargelegt haben, aber eben auf die absolute Aktualität nicht eingehen können. Hierzu bedarf es eines entsprechenden, die Schulbücher ergänzenden Zusatzmaterials. Aus der Perspektive der Lehrenden sind dies analoge wie digitale Medien, die von der lokalen über die überregionale Zeitung und wöchentliche Magazine bis hin zu YouTube-Videoclips oder Mitschnitten von Nachrichtensendungen reichen. Vielfach aber lassen auch solche hier angesprochenen potenziellen Unterrichtsmaterialien die Perspektive von direkt Betroffenen - und somit die Innenperspektive - eines aktuellen Ereignisses oder einer entsprechenden Expertin bzw. eines entsprechenden Experten vermissen, da sie letztlich mit einem medialen nicht pädagogischen Anliegen angefertigt und bereitgestellt werden.

Aus der Sicht des Globalen Lernens wäre diese Innenperspektive aber insofern von großer Bedeutung, da die Reproduktion von Stereotypen durch eurozentristische Perspektiven zu vermeiden und asymmetrischen Machtverhältnissen entgegen zu wirken ist (vgl. Danielzik, 2013, S. 26). Aufgrund ihrer oft globalen Netzwerke und vielfältigen Kontakte vor Ort sowie ihrer besonderen Expertise können an genau dieser Stelle von NRO verfügbar gemachte und eventuell gar bereits didaktisch aufbereitete (Unterrichts-)Materialien den entscheidenden Unterschied gegenüber anderen Anbietern von Informations- und Bildungsmaterialien ausmachen. 
Darüber hinaus bieten von NRO vorgelegte Bildungsmaterialien oftmals auch sach- und zeitgerechtere pädagogisch-didaktische Ansätze, die in die meist alle zehn bis fünfzehn Jahre neu gestalteten Bildungs- und (Rahmen-)Lehrpläne der Bundesländer noch nicht eingegangen sind. In der Vergangenheit ließ sich eine solche Diskrepanz bei umweltpädagogischen Ansätzen ebenso beobachten wie bei friedens-, entwicklungs- oder gesellschaftskritischen Bildungskonzepten. In jüngster Zeit wiederholt sich dieser Effekt grundsätzlich, wenngleich derzeit vor allem am Nachhaltigkeitsprinzip ausgerichtete, zukunftsorientierte Bildungskonzepte im Vordergrund stehen. Seit mit der Verabschiedung der „Agenda 21 “ und ihrem vielzitierten Kapitel 36 vor nunmehr drei Jahrzehnten „Bildung für nachhaltige Entwicklung“ als pädagogisch-didaktischer Ansatz entstand, entwickelt sich der fachliche Diskurs zu angemessenen Themen und didaktischen Prinzipien stetig weiter. Seit zwei Jahrzehnten lässt sich diese Entwicklung hinsichtlich der Präzisierung von als relevant erachteten spezifischen (B)NE-Kompetenzen beobachten, die Lernende zur aktiven Teilhabe an der „Großen Transformation“ hin zu einer nachhaltigen Gesellschaft entwickelt haben sollen (vgl. z.B. Menon \& Hoffmann 2012; Hoffmann 2016, Rieckmann 2018; Wiek et al. 2016). Von NRO bereitgestellte Bildungsmaterialien können aufgrund ihrer wesentlich kürzeren Entwicklungsphasen den Erkenntnisstand der fachlichen und didaktischen Debatte schon berücksichtigen bevor die Lehrpläne der Länder entsprechend angepasst und Schulbücher entwickelt werden konnten. So haben trotz der langen fachlichen wie didaktischen Diskussion (B)NE-Themen erst in den letzten fünf Jahren tatsächlichen Eingang in Bildungspläne und damit auch Schulbücher gefunden, etwa als "Leitperspektive BNE“ im Bildungsplan für allgemeinbildende Schulen in BadenWürttemberg.

Vor diesem Hintergrund wird deutlich, dass von NROs angebotene Bildungsmaterialien nicht nur unter dem Aspekt der Aktualität von Realereignissen, sondern auch unter dem Aspekt der Aktualität bildungskonzeptioneller Entwicklungen eine Vorreiterrolle einnehmen können. Darüber hinaus bieten sie häufig spezifische Perspektiven und stehen zeitnah zu aktuellen Ereignissen und zudem häufig kostenfrei zur Verfügung. Gleichwohl bedeutet dies aus schulischer Perspektive, dass Lehrkräfte die Vielzahl der etwa zum Thema Klimawandel aktuell vorliegenden Bildungsmaterialien der NRO (u.a. von GermanWatch e.V., Heinrich Böll-Stiftung, Greenpeace e.V., Klima Stiftung für Bürger/Klima Arena, Klimahaus Bremerhaven) sondieren und eigenständig prüfen, ggf. vor dem Hintergrund ihrer Lerngruppe anpassen müssen.

\section{Das Greenpeace Unterrichtsmaterial „Heiße Zeiten - Klima und Gesellschaft im Wandel": ein Fallbeispiel}

Eine der zahlreichen NRO, die in Schulen aktiv sind und Bildung mitgestalten, ist der gemeinnützige Verein Greenpeace e.V. Dieser nimmt seine Rolle als NRO auch im schulischen Kontext wahr und informiert durch seine Bildungsangebote insbesondere über globale ökologische und soziale Herausforderungen. Greenpeace e.V. ist laut Satzung international, überparteilich und völlig unabhängig von Politik, Parteien und
Industrie. Der „Zweck des Vereins ist die Forderung des Umwelt- und Tierschutzes sowie des Friedens und der Völkerverständigung. Greenpeace macht als international tätige ökologische Organisation die Probleme der Umwelt, insbesondere die globalen, bewusst und will so die Beeinträchtigung oder Zerstörung der natürlichen Lebensgrundlagen von Menschen, Tieren und Pflanzen verhindern. Greenpeace nimmt im Sinne des Umweltschutzes die Interessen der Verbraucher wahr“ (Greenpeace e.V. Satzung $₫ 2$ Zweck). Greenpeace e.V. bietet vielfältige Unterrichtsmaterialien, darunter auch didaktisch aufbereitete Unterrichtsvorschläge, an. Der Verein ist in der Lage, Unterrichtsmaterial innerhalb weniger Wochen nach einem aktuellen Ereignis zu erstellen und für die Schule nutzbar zu machen (https://www.greenpeace.de/bildung_bne).

Besonders wichtig und grundlegend bei der Erstellung dieser Unterrichtsmaterialien ist zum einen methodisch die Debatten- und Handlungsorientierung und zum anderen grundsätzlich der Beutelsbacher Konsens. Dieser fordert, kontroverse gesellschaftliche Debatten auch im Unterricht kontrovers und ergebnisoffen anzugehen und die Schülerinnen und Schüler nicht mit der dezidierten Meinung der Lehrenden gleichsam zu „überwältigen“ (Landeszentrale für politische Bildung Baden-Württemberg https://www.lpb-bw.de/beutels bacher-konsens). Insbesondere zielt die schulische Bildungsarbeit von Greenpeace e.V. neben der Informations- und Wissensvermittlung sowie des ,respektvollen und nachhaltigen Umgangs mit unseren Lebensgrundlagen“" (https://www.green peace.de/bildung_bne) darauf ab, die Handlungsfähigkeit, das Engagement sowie das politische Handeln der Lernenden im Hinblick auf Umweltschutz und nachhaltige Entwicklung im umfassenden Sinn zu stärken.

Die Fridays-for-Future-Bewegung hat das Thema Klimawandel und Klimakrise in die erste Reihe der gesellschaftlichen Diskussion gerückt und damit die Debatte belebt. Gute Debatten brauchen nicht nur Engagement, sondern vor allem eine argumentativ klare Grundlage. Hier setzt das Unterrichtsmaterial „Heiße Zeiten“ an. Wovon sprechen wir eigentlich beim Klimawandel? Was versursacht den Klimawandel? Gibt es tragfähige Lösungsansätze oder nur gute Ideen? Diese und viele andere grundlegende Fragen stehen daher im Mittelpunkt des schüleraktivierenden und zum kritischen Denken anregenden altersgerecht aufbereiteten Materials. Eine Reise durch Deutschland zum Thema Klimawandel und ein globaler Überblick zeigen, welche Auswirkungen der Klimawandel heute bereits aus der Perspektive der Ökologie (GREEN) und der potenziellen Konflikte (PEACE) zeigt. Der Klimawandel ist eine globale Herausforderung, die nur gemeinsam und uber Grenzen hinweg gemeistert werden kann. Gleichwohl bilden unsere natülichen Lebensgrundlagen und das friedliche, gerechte Miteinander die Voraussetzungen für das Leben auf der Erde. Diese werden durch den Klimawandel massiv verändert. Umweltschutz und Frieden sind unmittelbar miteinander verknüpft: Kein Umweltschutz ohne Frieden, kein Frieden ohne Umweltschutz. Mit diesem komplementären Perspektivwechsel von Umwelt und Frieden kann komplexen globalen Herausforderungen wie dem Klimawandel angemessen begegnet werden. Das Bildungsmaterial ist schulartubergreifend an die Bildungspläne der Länder für die Klassen 9-11 angelehnt, eignet sich insbesondere zur Kompetenzentwicklung in den 
Fächern Geographie, Politik und Gesellschaftswissenschaften und fördert den fächerübergreifenden Unterricht. Die in alle naturräumlichen wie gesellschaftlichen Sphären hineinreichenden Wirkungen des Klimawandels können durch interdisziplinäre Ansätze, wie sie in der Geographie verfolgt oder aber durch fächerübergreifende Unterrichtsansätze möglich werden, adäquat aufgegriffen und unterrichtlich thematisiert werden. Das Bildungsmaterial „Heiße Zeiten“ knüpft an den Nationalen Aktionsplan „Bildung für Nachhaltige Entwicklung“ an und möchte zur Umsetzung der „Agenda 2030“ mit ihren 17 Zielen für eine nachhaltige Entwicklung beitragen (online abrufbar: https://www.greenpeace.de/bildungsmateria lien).

\section{Kriterien der Erarbeitung von „Heiße Zeiten“}

Im Erstellungsprozess des Unterrichtsmaterials „Heiße Zeiten - Klima und Gesellschaft im Wandel“ wurden im reflexiven Prozess didaktische Kriterien diskutiert, die aus der Perspektive BNE/Globales Lernen von Relevanz sind. Das an der Erstellung beteiligte Redaktionsteam (Externer Autor, Vertreter*innen von Greenpeace e.V.) orientierte sich u.a. an (Beurteilungs-)Kriterien für Unterrichtsmaterialien des Welthauses Bielefeld (2007), educatión 21 (2012) sowie am Orientierungsrahmen für den Lernbereich Globale Entwicklung (2015). Tab. 1 legt die leitenden Kriterien dar, die bei der Entwicklung des Unterrichtsmaterials „Heiße Zeiten“ besondere Berücksichtigung fanden und im Folgenden näher beleuchtet werden. Anschließend folgt eine kritische Auseinandersetzung mit Herausforderungen beim Entstehungsprozess des Materials.

\section{Berücksichtigung der Kriterien im Entwick- lungsprozess des Materials „Heiße Zeiten“} Im Folgenden wird exemplarisch dargestellt, wie einzelne Bausteine des Unterrichtsmaterials gezielt angelegt wurden, um die in Tabelle 1 beschriebenen Kriterien zu berücksichtigen.

\section{Orientierung an aktuellen BNE-Bildungskonzepten und damit verbundenen Bildungszielen}

Bei der Erstellung dieses Bildungsmaterials war von Beginn an von großer Bedeutung, dass die Relevanz der „Agenda 2030“ und ein ganzheitliches, die ökologische, soziale, ökonomische, politische und kulturelle Perspektive berücksichtigendes Nachhaltigkeitsverständnis im gesamten Material deutlich zum Ausdruck kommen sollte. Diesen leitenden Kriterien folgend, wurde beispielsweise sowohl im Titel, als auch bereits im Vorwort des Materials signalisiert, dass es nicht allein um eine klimazentrierte Auseinandersetzung der Veränderungen geht, sondern um ein sich gegenseitig beeinflussendes Wechselspiel zwischen den Dimensionen, Umweltschutz und Frieden. Dabei ist nahliegend, dass der Terminus "Gesellschaft" sowohl politische als auch soziale, ökonomische und kulturelle Aspekte subsummiert. So wurde die Berücksichtigung aller genannten Nachhaltigkeitsdimensionen durch das gesamte Bildungsmaterial beibehalten. Dies kommt beispielsweise in der Darlegung von Umwelt und Frieden anhand dreier ausgewählter Fallballbeispiele aus unterschiedlichen Weltregionen (vgl. S. 4f.) ebenso zum Ausdruck, wie hinsichtlich der Formulierung der Arbeitsaufträge (Aktionismus oder gute Idee, S. 20f.) oder mit Blick auf die Auswahl bereits eingetretener Veränderungen im Zuge des Klimawandels.

\section{Orientierung an aktuellen BNE-Bildungskonzepten und} damit verbundenen Bildungszielen

- Kommt die Relevanz der „Agenda 2030“ im Rahmen des Bildungsmaterials zum Tragen?

- Liegt dem Bildungsmaterial sowie den Arbeitsaufträgen ein ganzheitliches (ökologisches, soziales, ökonomisches, politisches, kulturelles) Nachhaltigkeitsverständnis zugrunde?

- Werden die Kompetenzbereiche des Orientierungsrahmens „Erkennen“, „Bewerten“, „Handeln“ in Konzeption und Umsetzung adäquat berücksichtigt?

- Finden die von der UNESCO 2017 formulierten (B) NE-Schlüsselkompetenzen Berücksichtigung?

\section{Anschlussfähigkeit} an Schule

- Ist das Bildungsmaterial zielgruppenspezifisch konzipiert und gestaltet?

- Werden Möglichkeiten zur Vertiefung bzw. Weiterarbeit geboten?

- Ist die Anschlussfähigkeit des Bildungsmaterials an Bildungs- bzw. (Rahmen-)Lehrpläne gegeben?

- Werden bestehende Kontroversen der Fachdiskussion dargestellt?

- Sind die Lernziele benannt?

- Finden Operatoren Eingang in die Aufgabenkultur?

- Sind die Aktivitäten im Rahmen von Unterricht realisierbar (z.B. 45 od. 90 Minuten, ein Projekttag)?

\section{Methodisch-didaktische Gestaltung}

- Sind Handlungsoptionen angesprochen bzw. in der Aufgabenstellung berücksichtigt?

- Leistet das Bildungsmaterial mit seinen Arbeitsaufträgen einen Beitrag zur Stärkung des individuellen Engagements bzw. der individuellen Bereitschaft zur aktiven Teilhabe (z.B. in Gestalt politischen Engagements)?

- Ist die Aufgabenkultur durch eine angemessene Methodenvielfalt charakterisiert?

Multiperspektivität

- Wird das Thema mehrperspektivisch (z.B. Akteursperspektiven) behandelt?

- Wird der globale Kontext in der Darbietung deutlich?

- Werden Stereotype oder Diskriminierung in der Darstellung zugunsten einer objektiven Betrachtung vermieden? 
Ziel war es, die Kerndomänen des OR-Ansatzes (Erkennen, Bewerten, Handeln) sowie die von der UNESCO formulierten Schlüsselkompetenzen die im Rahmen einer Bildung für nachhaltige Entwicklung individuell zur Entfaltung kommen, in der Konzeption des Materials zu berücksichtigen. Gelöst wurde dies beispielsweise auf den beiden Doppelseiten (S. 6-9) „Klimawandel - wovon sprechen wir eigentlich?“. Hier sollen die Schüler*innen die klimarelevanten physikalischen Faktoren und Prozesse erkennen, die zur Ausprägung des aktuellen Klimas führen. Komplementär hierzu soll mit der Aufgabe „Analysiere die verschiedenen Faktoren, die Einfluss auf unser Klima haben. Dazu stehen dir die Informationstexte und Grafiken zur Verfügung “ die Kompetenz zum systemischen Denken gestärkt werden.

Für Konzipierung der Doppelseite „Eine gute Idee oder wirkungsloser Aktionismus“ (S. 20f.) sollten die Kerndomäne Bewerten und insbesondere die Kompetenz zum vorausschauenden Denken leitend aufgegriffen werden. Schüler*innen werden aufgefordert, begründete Stellungnahmen zu den gebotenen diversen Beispielen zu beziehen, inwieweit die Lösungsansätze einen Beitrag zur Minderung der Erderwärmung leisten. Handlungsoptionen und damit eine im Schulalltag oft nur schwer erreichbare Handlungsebene wird insbesondere beim Thema "Meine Schule für den Klimaschutz" (S. 28f.) hervorgehoben. Die konkrete handlungsorientierte Aufgabenstellung entlang eines Leitfadens, die eigene Schule näher zu beleuchten und den „Klima-Fußabdrucks“ an der eigenen Schule durch selbst entwickelte Maßnahmen zu reduzieren, greift auf die Idee des Whole School Approaches zurück. Schulgebäude zählen schließlich zu den größten Energieverbrauchern der öffentlichen Hand.

Mit der Senkung des „Klima-Fußabdrucks“ an der eigenen Schule durch selbst entwickelte Maßnahmen kann dann ein konkreter Beitrag zum Klimaschutz geleistet werden. Mit diesem Impuls zum konkreten Handeln werden insbesondere die Kompetenzen zum kritischen Denken sowie zur Problemlösung betont, indem die Schüler*innen am konkreten Beispiel der eigenen Schule differenziert eigene Handlungsoptionen entwickeln sollen.

\section{Methodisch-didaktische Gestaltung}

Ziel war es, bei der methodisch-didaktischen Gestaltung des Materials dem Anspruch einer Methodenvielfalt gerecht zu werden. Dies wurde insbesondere durch den Aufbau des Gesamtmaterials realisiert: die Methodenvielfalt ist durch die Kombination von Arbeitsseiten, Arbeitsaufträgen und Operatoren auf unterschiedlichen Anforderungsbereichen gegeben.

Sie reicht von der fragengeleiteten Auswertung von Texten und unterschiedlichen Grafiken, der Verwendung einer Analysematrix zu den verschiedenen Faktoren, die Einfluss auf unser Klima haben (S. 6-9) über die Bewertung unterschiedlicher Maßnahmen und Aktionen zum Klimaschutz (S. 20 f.) bis hin zur Herausbildung einer eigenen Position zum Klimaschutzpaket der Bundesregierung (S. 18f.) oder der Planung und Organisation einer themengebundenen Reise zu Orten des Klimawandels in Deutschland (S. 26f.). In der Kombination dieser Aspekte wird der spezifische didaktische Mehrwert des Bildungsmaterials manifest.

\section{Anschlussfähigkeit an Schule}

Bestehende Kontroversen mit Blick auf den Klimawandel kommen im Rahmen der Pariser Klimabeschlüsse (S. 17) ebenso zum Tragen wie am Beispiel der Stellungnahme verschiedener Gruppierungen und Verbände zum Klimapaket (S. 18f.), womit eine vielperspektivische und diskursive Darstellung gewährleistet werden soll. Eine Darlegung der Position der sogenannten Klimaskeptiker ist jedoch als Quellenmaterial bewusst nicht aufgenommen worden, da eine solche Debatte weit über das hier für die Schüler*innen und aufbereitete Material hinausgehen würde. Die Auseinandersetzung mit dieser Position kann bei Bedarf jedoch mittels der angegebenen Internetseite „Klimafakten.de“ angebahnt werden.

Mit dem Ziel, die Anschlussfähigkeit an Schule zu gewährleisten, wurden die Arbeitsaufträge überwiegend unter Verwendung von Operatoren aus unterschiedlichen Leistungsbereichen formuliert und entsprechen somit der Konzeption kompetenzorientierten Unterrichtens, wie beispielsweise in den folgenden Aufgaben: „Stelle Bezüge der Beispiele Anden, Tschad oder Lausitz zu den 17 Zielen für nachhaltige Entwicklung dar (siehe S. 4 und 5). Gibt es Zielkonflikte?“ (S. 25) oder „Formuliert einen Titel für eure Reise zum Thema Klimawandel. Formuliert einen programmatischen, motivierenden Ankündigungstext" (S. 27).

Der Einsatz von W-Fragen sollte das Verständnis aus Schülerperspektive verbessern und einen stärkeren Aufforderungscharakter einbringen. So wurden einzelne Aufgabenformulierungen entlang von W-Fragen strukturiert, wie beispielsweise auf S. 8: „Was sagen Bürgermeister*innen, was die Parteien? Was sagen Umwelt- und Entwicklungsorganisationen?".

\section{Multiperspektiven}

Für die Auseinandersetzung mit dem komplexen, globalen Thema Klimawandel bedarf es der Berücksichtigung diverser Perspektiven, von global bis lokal, aus ökologischer oder sozialer, aber auch aus ökonomischer sowie politischer Perspektive, als Individuum, Kommune oder Staat. Diese sollten konsequent in „Heiße Zeiten - Gesellschaft und Klima im Wandel“ zum Ausdruck kommen. Klimawandel und die existenzielle Bedrohung für Menschen werden dafür aus ökologischer und friedenspolitischer bzw. gesellschaftlicher Perspektive näher beleuchtet (S. 4f.) oder auch das Pariser Klimaschutzabkommen sowohl aus der Perspektive der politischen Entscheidungsträger sowie aus der Perspektive der F4F-Bewegung thematisiert (S. 16f.). Insbesondere wurden die Perspektiven verschiedener gesellschaftlicher Gruppen und Verbände am Beispiel klimapolitischer Beschlüsse der Bundesregierung, die Ende 2019 Eingang in das sogenannte „Klimapaket“ fanden, bewertet (S. 18f.). Auch die auf S. 30 gelisteten Literatur- und Internethinweise erweitern das Perspektivenspektrum auf freiwilliger Basis um weitere Diskutanten und Gruppierungen.

Die globale Perspektive wurde bei der Konzipierung des Materials als leitendes Kriterium genannt. Der globale Kontext kommt deswegen an vielen Stellen des Bildungsmaterials zum Tragen, wie beispielsweise bei der Auswahl der konfliktträchtigen Wirkung klimawandelbedingter Veränderungen in Deutschland, am Tschadsee und in den Anden (S. 4f.). Die Seiten (S. 22f.) „Heiße Zeiten - weltweit spürbar“ sind explizit 
global ausgerichtet und führen anhand vielfältiger Beispiele in die bereits eingetretenen Veränderungen des Weltklimasystems ein. Auch die SDG-Perspektive unterstreicht die globale Dimension der Herausforderung sowie eines notwendigen global-gesellschaftlichen Lösungsansatzes (S. 24f.).

Herausforderungen Interessensvertretung vs. Neutralität? Bei der Erstellung des Unterrichtsmaterials „Heiße Zeiten Klima und Gesellschaft im Wandel“" sind das Bildungsteam von Greenpeace e.V. mit dem Autor des Materials auf Herausforderungen gestoßen. Zwischen Interessensvertretung und angemessenem und gefordertem Maße an Neutralität aufgrund des Beutelsbacher Konsens, welcher - so betont Prof. Bernd Overwien (2016) - kein Neutralitätsgebot sei, tat sich ein Spannungsverhältnis auf, mit welchem es umzugehen galt. Dieses kristallisierte sich z.B. an der Überschrift der Doppelseiten 14 und 15. „Warum ist der Klimawandel so gefährlich?“. So ließe sich am kleinen Wörtchen „so“ aus der Überschrift eine Bewertung ablesen, die den Schüler*innen bei der Stärkung ihrer Kompetenz des Bewertens vorgreifen könnte. Demgegenüber stand die Argumentation, dass bei den gewählten Blickpunkten der Auswirkungen des Klimawandels und deren Dimensionen, wie z.B. Artensterben, Klimafolgekosten, Konflikte durch Hunger und Migration oder Kippelemente im Klimasystem, das Wort „so“ in der Überschrift durchaus gerechtfertigt sei, um Ernst und Tragweite der Situation zu verdeutlichen. Zugleich signalisiert der Einbezug des Wortes bereits eine Wertung und dokumentiert somit den Konflikt zwischen politischer Positionierung und sachorientierter Neutralität.

Eine ähnlich gelagerte Diskussion zwischen Klima- und Bildungsexpert*innen ging der ausführlichen Darstellung des natürlichen Klimawandels in dem Bildungsmaterial voraus: Warum, so die Frage, sollte den natürlichen Ursachen des Klimawandels in dem Material verhältnismäßig viel Raum gegeben werden? Über $90 \%$ der internationalen Klimaforscher*innen sind der Überzeugung, dass der heutige fortschreitende Klimawandel in seinen Dimensionen überwiegend anthropogen - von Menschen - verursacht ist. Dennoch wurde bei der Frage, „Klimawandel - wovon sprechen wir eigentlich" (S. 6 f.) umfangreich und kontrovers auch auf die Ursachen natürlichen Klimawandels eingegangen, um den Schüler*innen ein physikalisches Verständnis hierüber zu ermöglichen und den Anteil natürlicher und anthropogener Ursachen des Klimawandels selbstständig zu bewerten.

Basierend auf klimawissenschaftlichen Analysen, wie etwa denen des Weltklimarates (IPCC), besteht kein Zweifel, dass der anthropogene Klimawandel zur aktuellen Klimakrise geführt hat. Die vorliegenden wissenschaftlichen Belege sind eindeutig und dokumentieren, dass die aktuelle Klimakrise mit ihren weltweiten Auswirkungen (u.a. Dürre, Extremwetter, Anstieg des Meeresspiegels) sehr gefährlich für das Leben auf der Erde ist. Dies gilt für Menschen, Tiere und Pflanzen gleichermaßen. Deshalb setzt sich Greenpeace in öffentlichen Diskussionen sowie politischen Forderungen für wirksamen und ambitionierten Klimaschutz ein. In der Bildungsarbeit von Greenpeace wird insbesondere darauf Wert gelegt, dass Schüler*innen ausreichend über die Ursachen und Auswirkungen informiert sind, alternative klimafreundliche Handlungsoptionen kennenlernen und diese auch in ihrem Alltag sowie spä- terem beruflichen Leben verfolgen können. Im Kontext eines emanzipatorischen Bildungsverständnisses ist es jedoch gleichermaßen wichtig, sich auch mit den unterschiedlichen Faktoren der Klimaveränderungen analytisch und kritisch-reflektierend auseinanderzusetzen, um eigene Urteile und Bewertungen entwickeln zu können. Nur die differenzierte fachliche Durchdringung klimarelevanter Faktoren und Prozesse schafft ein ausreichendes Verständnis, um Fehlinterpretationen von sogenannten „Klimaskeptikern“ zu erkennen, auf der Basis naturwissenschaftlicher Logik zu entlarven und entsprechend dagegen argumentieren zu können.

\section{Fazit und Ausblick}

In diesem Beitrag wurde exemplarisch am Unterrichtsmaterial „Heiße Zeiten - Klima und Gesellschaft im Wandel“ von Greenpeace e.V. sowie entlang von ausgewählten Kriterien diskutiert, inwieweit von gemeinnützigen Nichtregierungsorganisationen (NRO) erstellte Bildungsmaterialien einen Mehrwert für Schüler*innen darstellen können. Dabei wurde herausgearbeitet, dass NRO sich oftmals besonders durch die spezifische Innenperspektive, Aktualität der Thematik und innovative didaktisch-methodische Ansätze hinsichtlich gesellschaftlich relevanter Themen auszeichnen, die mittels entwickelter Unterrichtsmaterialien und -impulse Eingang in den schulischen Alltag finden können. Darin liegt zum einen eine Chance, zum anderen aber auch die Herausforderung Anschlussfähigkeit für Schule und Unterricht zu schaffen, wie z.B. durch eine Operatoren Konformität, Bezüge zu Lehrplänen oder auch wissenschaftspropädeutischem Unterricht, der nicht zwangsläufig und prioritär im Erfahrungsbereich und der Expertise einer NRO liegt. Vor allem letzteres unterstreicht die Empfehlung, Unterrichtsmaterialien in Zusammenarbeit mit Didaktiker*innen zu erstellen. Der Beitrag macht deutlich, dass von NRO entwickeltes Unterrichtsmaterial durchaus eine sinnvolle Ergänzung sowie einen Mehrwert für Schüler*innen bieten kann, solange die im Beitrag genannten Kriterien berücksichtigt werden. Am konkreten Beispiel „Heiße Zeiten - Klima und Gesellschaft im Wandel“" von Greenpeace e.V. beruht dieser Mehrwert insbesondere auf der Aktualität der dargestellten Entwicklungen sowie auf dem spezifischen Blickwinkel, Ökologie bzw. naturräumliche Veränderungen dezidiert mit Friedensaspekten in Verbindung zu bringen. Dies ist in vorliegenden Lehrbüchern zwar ansatzweise berücksichtigt, aber in der Regel nicht mit dieser starken Akzentuierung.

\section{Literatur}

Böhme, L. (2019). Politische Bildung für Schülerinnen und Schüler mit sonderpädagogischem Förderbedarf. Perspektiven Globalen Lernens an Förderzentren. Frankfurt am Main: Wochenschauverlag.

Danielzik, C. (2013). Postkoloniale Perspektiven auf Globales Lernen und Bildung für nachhaltige Entwicklung. Zeitschrift für internationale Bildungsforschung und Entwicklungspädagogik, 13(1), 26-33.

education 21 (2020): Qualitätskriterien | Evaluation von Unterrichtsmedien | Globales Lernen \& BNE. Zugriff am 20.05.2020 https://www.education21.ch/sites/default/files/uploads/pdf-d/Kriterienkataloge_Medienevaluation_GL\%26UB.pdf

Greenpeace e.V. (2020): Heiße Zeiten - Klima und Gesellschaft im Wandel. Zugriff am 20.04.2020 https://www.greenpeace.de/sites/www.greenpeace.de/files/gpbm bildungsmaterial_heisse_zeiten.pdf 
Greenpeace e.V. (2020): Greenpeace e.V. zu Bildung für Nachhaltige Entwicklung. Zugriff am 20.04.2020 https://www.greenpeace.de/bildung_bne

Hoffmann, T. (2016). Welche Kompetenzen für eine Bildung für nachhaltige Entwicklung brauchen Lehrende? Lehren \& Lernen, 16(8/9), 34-38.

Landeszentrale für politische Bildung Baden-Württemberg: Der Beutelsbacher Konsens. Zugriff am 01.06.2020 https://www.lpb-bw.de/beutelsbacher-konsens

Menon, S. \& Hoffmann T. (2012). Out of the confusion of E-S-and D-competences. Zugriff am 01.06.2020 http://www.esd-expert.net/files/ESD-Expert/pdf/Introduc tion_3_Out-of-the-confusion.pdf

Möller, J. (2009). Kooperation mit entwicklungspolitischen NRO im Politikunterricht und im Fach Sozialwissenschaften. Zeitschrift für internationale Bildungsforschung und Entwicklungspädagogik, 32(1), 27-30.

Overwien, B. (2016) Globales Lernen und politische Bildung - eine schwierige Beziehung? Zeitschrift für internationale Bildungsforschung und Entwicklungspädagogik, 39(2), 7-11.

Rieckmann, M. (2018). Chapter 2 - Learning to transform the world: key competencies in ESD. In A. Leicht. Heiss \& W.J. Byun (Hrsg.), Issues and trends in Education for Sustainable Development (S. 39-59). Paris: UNESCO. Zugriff am 02.05.2020 https://unesdoc.unesco.org/ark:/48223/pf0000261802

UNESCO (2017). Education for Sustainable Development Goals: learning objectives. Zugriff am 20.04.2020 https://unesdoc.unesco.org/ark:/48223/pf0000247444

Welthaus Bielefeld (2007). Beurteilungskriterien von Unterrichtsmaterialien für das „Globale Lernen“" Hrsg. v. Welthaus Bielefeld. Zugriff am 20.05.2020 http://doku. cac.at/0709_pw_beurteilungskriterien_ums_globales_lernen.pdf
Wiek, Arnim et al. (2016). Operationalising Competencies in Higher Education for Sustainable Development. In: M. Barth, G. Michelsen, M. Rieckmann, I. Thomas (Hrsg.), Routledge Handbook of Higher Education for Sustainable Development (S. 241-260). London \& New York: Routledge.

Zivilgesellschaft in Zahlen (ZiviZ) (2017). Vereine, Stiftungen und Co: Die Neuen Bildungspartner? Sonderauswertung des ZiviZ-Surveys 2017. o.O: o.V.

\section{Katarina Roncevic}

verfügt über mehr als 8 Jahre Erfahrung in den Bereichen BNE, inklusive Bildung und Whole School Approach. Seit 2019 arbeitet sie für Greenpeace Deutschland im Bildungsteam und ist Doktorandin an der Universität Vechta. Sie ist Mitglied des internationalen ESD Expert-Net.

\section{Dr. Thomas Hoffmann}

Fachleiter Geographie am Studienseminar Karlsruhe, Lehrbeauftragter für Geographiedidaktik am Karlsruhe Institut für Technologie (KIT), Gymnasiallehrer für die Fächer Geographie, Geschichte, Politik und Wirtschaft, Gründungsmitglied des ESD Expert Net.

\section{UNSERE BUCHEMPFEHLUNG}

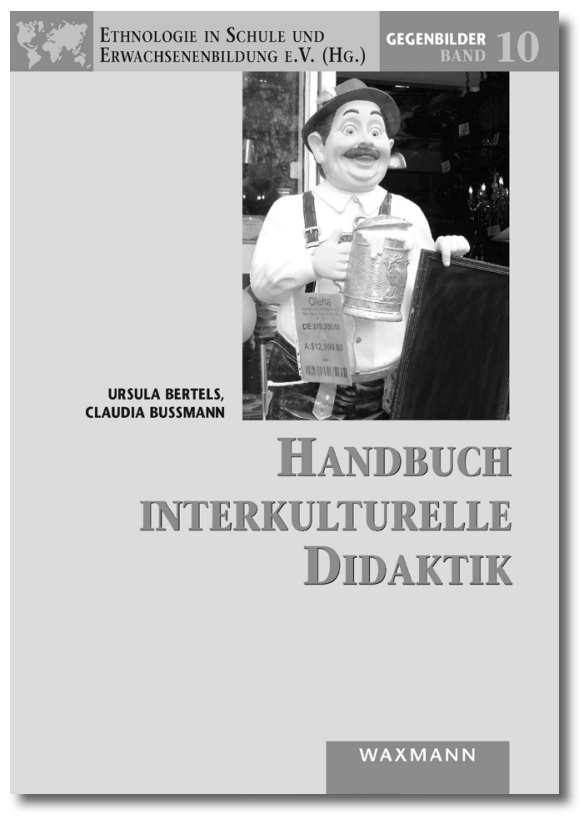

Gegenbilder, Band 10 2020, 2., überarbeitete Auflage, 236 Seiten, br., $24,90 €$ ISBN 978-3-8309-4212-2

E-Book: 21,99

ISBN 978-3-8309-9212-7
Claudia Bußmann, Ursula Bertels

\section{Handbuch interkulturelle Didaktik}

Nur durch interkulturelles und globales Lernen ist es möglich, Kindern und Jugendlichen interkulturelle Kompetenz zu vermitteln und sie damit auf ein Leben in einer multikulturellen Gesellschaft auf globaler und lokaler Ebene vorzubereiten.

Doch was ist Interkulturelle Kompetenz überhaupt? Welche Lernziele interkulturellen und globalen Lernens gibt es? Und wie lassen sich diese im Unterricht umsetzen?

Ziel des Handbuchs Interkulturelle Didaktik ist es, die theoretischen Hintergründe des interkulturellen und globalen Lernens sowie die praktischen Umsetzungsmöglichkeiten aufzuzeigen. Obwohl der Schwerpunkt des Handbuchs auf der Schule liegt, lassen sich die vorgestellten Methoden und Materialien in angepasster Form auch in der Erwachsenenbildung einsetzen. 